\title{
ESTUDO DE VÁLVULAS ARTIFICIAIS NO CÓLON ESQUERDO APÓS AMPUTAÇÃO ABDOMINOPERINEAL PARCIAL DO RETO MAIS COLOSTOMIA PERINEAL, EM CÃES
}

\author{
Sávio Lana SIQUEIRA ${ }^{1}$, Alcino LÁZARO-da-SILVA², \\ Otávio Augusto Fonseca REIS ${ }^{3}$, Rodrigo Santana FANTAUZZI ${ }^{3}$, \\ Otávio de Melo SILVA Jr. ${ }^{3}$ e Paulo Guilherme de Oliveira SALES ${ }^{3}$
}

\begin{abstract}
RESUMO - Racional - Ao longo do tempo, estudou-se a escolha da colostomia perineal como a forma de se evitar a colostomia abdominal após cirurgia de amputação abdominoperineal. A colostomia perineal associada a um mecanismo frenador das fezes vem sendo estudada há vários anos com resultados satisfatórios. Objetivo - Investigação, em cães, da valvuloplastia cólica aplicada à amputação abdominoperineal do reto mais colostomia perineal e a discussão dos resultados obtidos na avaliação clínica e histopatológica. Material e Métodos - O modelo experimental desta pesquisa foi desenvolvido no cólon de cães. Foram operados 65 animais, dos quais 10 constituíram o grupo-piloto, 15 o grupo-controle e os 40 demais foram separados em três grupos. O grupo 1 para observação de 10 dias; o grupo 2 em pós-operatório de 20 dias; e o grupo 3 de 30 dias. Os cães foram submetidos a amputação abdominoperineal parcial do reto com colostomia perineal associada à seromiotomia circunferencial, extramucosa em cólon esquerdo (exceto nos dos grupos-controle). Resultados - Através de acompanhamento e estudo post-mortem, foi possível verificar fezes sólidas a montante das válvulas, evidenciando-se o eficiente mecanismo frenador das mesmas. A análise histológica das válvulas mostrou neoproliferação conjuntiva com fibrose em toda a área seccionada da serosa e das camadas musculares, separando-as. Evidenciou-se um anel fibroso com diminuição do lúmen intestinal, em resposta à cicatrização da serosa e muscular invertidas pela sutura, levando à formação da "válvula artificial". O processo inflamatório fibrosante reparativo ocorreu em todas as válvulas, associado a reações do tipo corpo estranho e à estenose parcial do lúmen intestinal. Conclusão - Conclui-se que a seromiotomia circunferencial produz um anel fibroso que facilita a estase fecal em colo esquerdo.
\end{abstract}

DESCRITORES - Colostomia. Reto, cirurgia. Cães.

\section{INTRODUÇÃO}

A amputação abdominoperineal, com a exérese do reto e ânus, como preconizado por MILES et al..$^{(16)} \mathrm{em}$ 1908, leva à colostomia abdominal e ao uso de bolsa abdominal coletora.

Ao longo do tempo estudou-se a escolha da colostomia perineal como forma de se evitar a abdominal. Por ser essa desprovida de um esfíncter, vários autores descreveram métodos de retenção das fezes, promovendo a substituição do conjunto esfincteral por retalhos e rotações musculares, alguns sendo até estimulados eletricamente e por dispositivos mecânicos valvulares ${ }^{(1,2,3,7,8)}$.

Com o mesmo objetivo de se evitar a colostomia abdominal, LÁZARO-da-SILVA, em $1991^{(10)}$, realizou a colostomia perineal valvulada. Propôs a realização de duas ou três seromiotomias extramucosas: a primeira mais ou menos $10 \mathrm{~cm}$ acima da estomia no períneo; as outras, também, a cerca de $10 \mathrm{~cm}$ uma da outra, proximalmente, à primeira. Dessa forma, obtém-se retardo ou mecanismo

${ }^{1}$ Disciplina de Anatomia da ${ }^{3}$ Faculdade de Ciência de Minas Gerais; ${ }^{2}$ Disciplina de Cirurgia do Aparelho Digestivo da Faculdade de Medicina da Universidade Federal de Minas Gerais, Belo Horizonte, MG.

Endereço para correspondência: Dr. Sávio Lana Siqueira - Av. Pasteur, 89 - sala 1605 - Santa Efigênia - 3015-0290 - Belo Horizonte, MG. E-mail: saviosiqueira@ig.com.br 
frenador das fezes no intestino grosso. Houve boa aceitação dessa colostomia valvulada, que melhorou a qualidade geral de vida dos pacientes.

A colostomia abdominal definitiva nem sempre é mais aceitável que a neoplasia em si, ou mesmo a sua recidiva. Para evitá-la, no tratamento do adenocarcinoma reto-anal, é necessária grande margem de segurança, o que aumentaria a radicalidade da operação. O tratamento mais seguro seria a cirurgia, suplementada pela radioterapia, quimioterapia e imunoterapia ${ }^{(10,11,12,13)}$.

Neste estudo, essa proposta denominou-se valvuloplastia cólica e foi colocada no nível experimental, em cães, para análises clínicas e histopatológicas da sua evolução pós-operatória.

O objetivo deste estudo foi a investigação, em cães, da valvuloplastia cólica aplicada à amputação abdominoperineal parcial do reto mais colostomia perineal e a discussão dos resultados obtidos na avaliação clínica e histopatológica.

\section{MÉTODO}

O modelo experimental desta pesquisa foi desenvolvido no cólon de cães, pela semelhança anatômica de seu intestino com o humano.

Os animais foram tratados conforme a Lei $\mathrm{n}^{\circ} 6.638$, de 8 de maio de 1979, Decreto $n^{\circ} 24.645$ de 10 de julho de 1934, e as normas internacionais de bons tratos aos animais, conforme literatura pesquisada e de acordo com os princípios éticos do Colégio Brasileiro de Experimentação Animal (COBEA) ${ }^{(17,18)}$.

Foram utilizados 65 animais, 37 machos e 28 fêmeas, de porte médio, cedidos pelo canil do Setor de Zoonoses da Prefeitura Municipal de Belo Horizonte. Desses, 10 foram submetidos a um estudo-piloto, sem observação pós-operatória: o grupo piloto.
Quinze foram operados, constituindo-se os grupos-controle: um, com cinco animais observados por 10 dias; outros cinco, por 20 dias, e mais cinco observados por 30 dias, constituindo-se, respectivamente, os grupos-controle 1,2 e 3 .

Os outros 40 animais foram operados e separados em três grupos. O grupo 1 (de 14 cães, 11 machos e 3 fêmeas) para observação pós-operatória de 10 dias; o grupo 2 (13 cães, 6 machos e 7 fêmeas), em pós-operatório de 20 dias; e o grupo 3 (de 13 cães, 5 machos e 8 fêmeas) de 30 dias.

Os cães foram submetidos a uma laparotomia mediana infra-umbilical (fêmeas) ou longitudinal transretal infraumbilical à esquerda (machos), seguindo-se de exploração da cavidade peritonial. Não se constataram ausência de alterações macroscópicas viscerais e vasculares que pudessem prejudicar o trabalho. Todo o cólon descendente e parte superior do reto foram mobilizados, preservando-se a irrigação pela artéria mesentérica caudal e arcada paracólica. Foi identificado e preservado o linfonodo mesentérico caudal, na altura da bifurcação da artéria mesentérica caudal. A seguir, feito descolamento, por divulsão romba, do reto.

Foi realizada seromiotomia circunferencial, extramucosa, no cólon descendente, no nível do linfonodo mesentérico caudal, outra no mesmo cólon, porém mais cranial e uma outra no reto intraperitonial. Essas seromiotomias distanciavam-se de $7 \mathrm{~cm}$ a 10 cm uma da outra, tendo como indicação de seromiotomia completa a constatação visual clara da protrusão da mucosa.

A sutura sobre a área de mucosa exposta da seromiotomia foi feita em toda a circunferência do segmento intestinal, com pontos simples, extramucosos, de fio de seda agulhada 4-0, criando-se uma válvula, intraluminar, circunferencial (Figura 1).

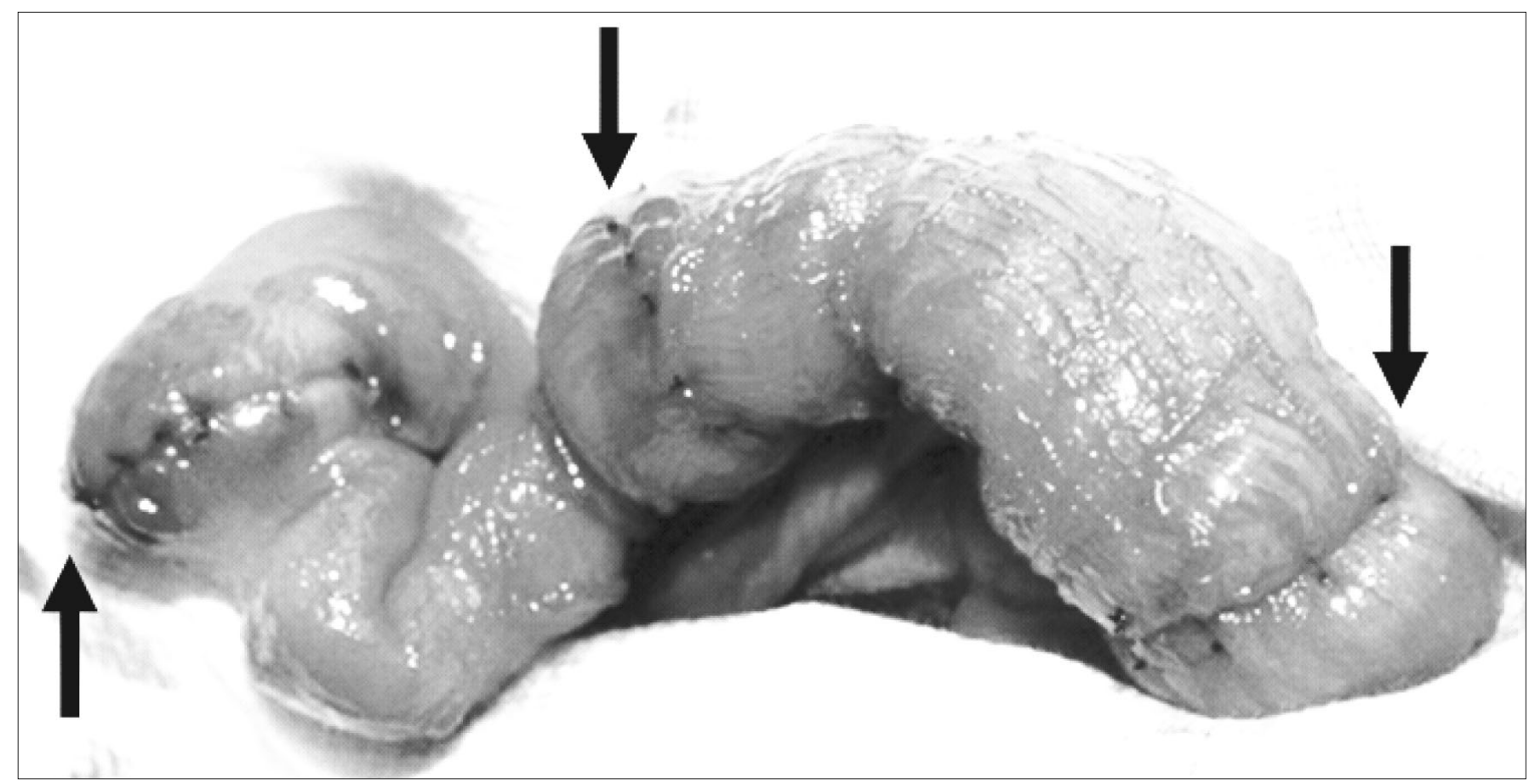

FIGURA 1 - Suturas sobre a área da mucosa exposta após as três seromiotomias transversais, circunferenciais, extramucosas, constituindo as válvulas 
Passando para o tempo operatório perineal, manteve-se o cão em decúbito dorsal, porém com os membros posteriores elevados, com exposição do períneo. Realizou-se: incisão perineal circular da pele e subcutâneo, a $1 \mathrm{~cm}$ da margem ano-cutânea; incisão circular do assoalho pélvico, promovendo a retirada em bloco da porção perianal e perirretal dos músculos levantadores do ânus; ligadura do pedículo vascular retal inferior na intimidade dos mesmos músculos; liberação do reto, tração caudal e amputação de $2 \mathrm{~cm}$ caudais do mesmo incluindo-se o bloco de pele, subcutâneo e musculatura; colostomia no orifício cirúrgico perineal, com maturação primária, pela sutura do reto ao subcutâneo e pele com 12 pontos separados, circunscritos, de categute agulhado 2-0 simples.

Seguiu-se a liberação do animal para recuperação operatória em baia individual, estando a dieta de água e ração já liberada. Os 15 cães do grupo-controle foram submetidos ao mesmo ato operatório descrito, porém, não se realizou o tempo operatório das seromiotomias transversais, circunferenciais e extramucosas.

Todos os cães foram acompanhados e avaliados diariamente, levando-se em conta a data de reinício espontâneo de ingestão de água e ração; feitos exame clínico, exame da ferida operatória abdominal e da colostomia perineal, com a realização de toque retal, no segundo dia pós-operatório. As evacuações foram observadas diariamente e as fezes analisadas quanto ao estado de hidratação, consistência e formato.

Os cães foram necropsiados após o período de observação. Realizouse: relaparotomia mediana supra e infra-umbilical, identificação da região onde foram feitas as seromiotomias e as válvulas, a adesiólise do segmento cólico, exérese de todo o intestino grosso, abertura longitudinal do mesmo, com incisão contínua em sua face ventral, esvaziamento e lavagem da mucosa com água corrente.

De cada cão necropsiado dos grupos 1, 2 e 3, retirou-se um pequeno segmento da válvula situada no nível do linfonodo mesentérico caudal, e imediatamente levado para o laboratório de histopatologia.

Um cão de cada grupo foi escolhido, aleatoriamente, e submetido a colonoscopia para evidenciar-se melhor o aspecto final das válvulas ainda com o cão vivo.

\section{RESULTADOS}

\section{Controle morfológico das fezes}

Foram realizadas análises comparativas dos grupos de estudo, quanto ao grau de controle morfológico das fezes (muco, líquidas, pastosas ou sólidas) ao final do período de observação de cada grupo (Graficos 1, 2, 3).

Nos grupos 1, 2 e 3 houve cães que evacuaram fezes sólidas.

\section{Toque retal}

No segundo dia pós-operatório procedeu-se, em todos os cães dos grupos 1, 2, 3 e nos de controle, o toque retal para análise da vitalidade do segmento intestinal abaixado. Em todos os casos, o reto apresentou-se viável, com consistência e tônus da musculatura lisa inalterados. Onze cães dos 40 operados nos grupos 1,2 e 3 (27,57\% dos casos) apresentaram fezes no reto remanescente. Em 29 cães (72,5\% dos casos) não se observaram fezes ao toque retal. Dos três grupos-controle, em 5 animais,
GRÁFICO 1 - Análise descritiva do grupo 1 e grupo-controle 1, quanto ao grau do controle morfológico das fezes

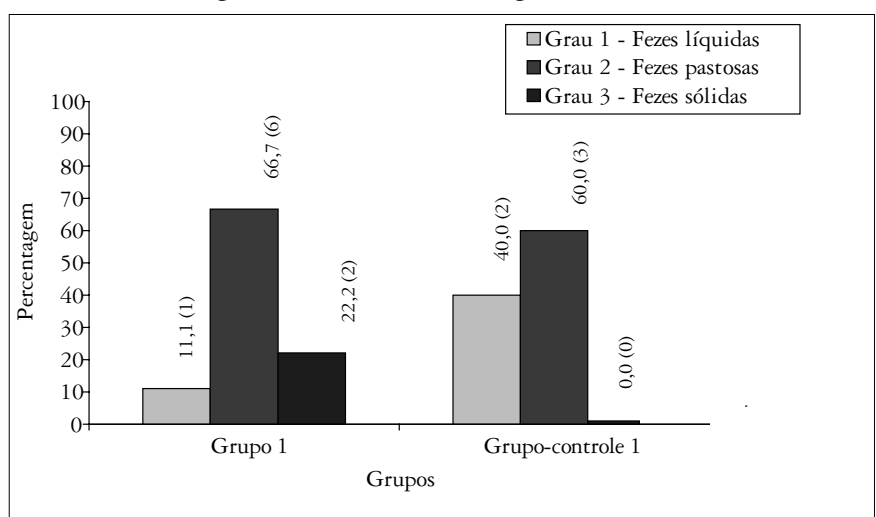

GRÁFICO 2 - Análise descritiva do grupo 2 e grupo-controle 2, quanto ao grau do controle morfológico das fezes

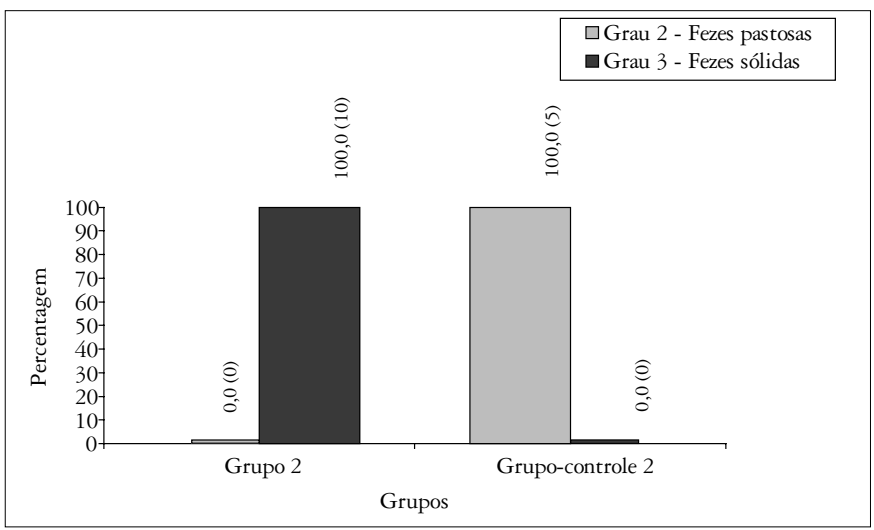

GRÁFICO 3 - Análise descritiva do grupo 3 e grupo-controle 3, quanto ao grau do controle morfológico das fezes

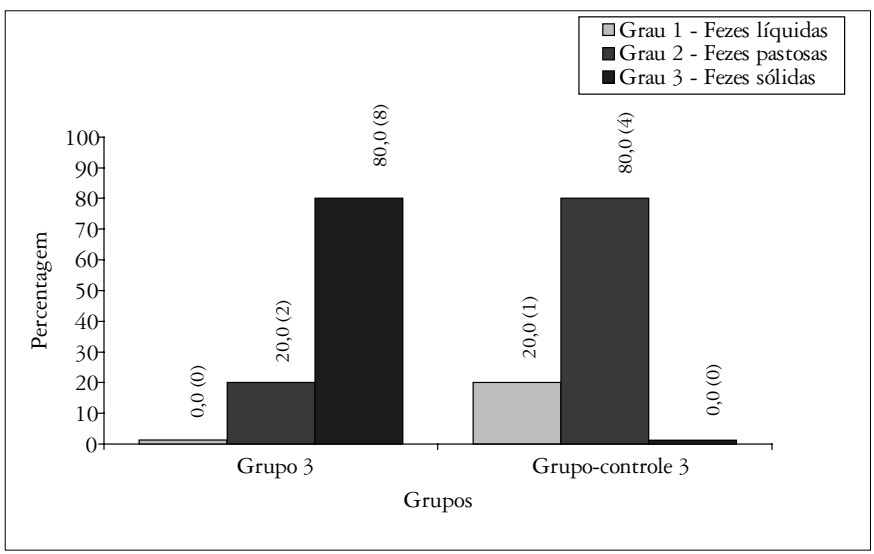

fezes estavam presentes ao toque retal $(33,3 \%)$, porém nos outros 10 cães o reto estava sem fezes $(67,7 \%$ dos casos $)$.

Considerando-se o grupo 1, dos nove cães que sobreviveram ao período de observação, sete $(77,8 \%)$ não apresentaram fezes ao toque e em dois $(22,2 \%)$ houve presença de fezes. No grupo- 
controle 1 , dos cinco animais, três $(60 \%)$ não apresentavam fezes ao toque, dois $(40 \%)$ as tinham no reto.

No grupo 2, dos 10 cães que sobreviveram ao período de observação, 5 (50\%) não apresentaram fezes ao toque; em outros 5 cães $(50 \%)$ houve presença de fezes. No grupo-controle 2 , dos cinco animais, em quatro (80\%) não se observaram fezes ao toque, um cão $(20 \%)$ apresentou fezes ao toque.

No grupo 3, dos 10 cães que sobreviveram ao período de observação, 9 (90\%) não apresentaram fezes ao toque, um cão (10\%), sim. No grupo-controle 3, dos 5 animais, em $3(60 \%)$ o reto estava sem fezes, dois cães $(40 \%)$ as apresentaram.

\section{Necropsia}

Nas necropsias de todos os cães, que neste estudo apresentaram evacuações com fezes sólidas, observaram-se dilatações, a montante das válvulas, indicando estase fecal e o mecanismo frenador possibilitado pelas mesmas.

\section{Colonoscopia}

Nas colonoscopias realizadas, evidenciou-se haver a constrição e redução do lúmen intestinal, caracterizando a formação de uma válvula muscular revestida internamente de mucosa.

\section{Análise histológica}

Após a necropsia dos cães, realizou-se a exérese de todo o intestino grosso. Procedeu-se, aleatoriamente, a escolha de cinco cães de cada grupo de estudo operado (com válvula), fez-se biopsia incisional, em fita de $1 \mathrm{~cm}$ de largura e $3 \mathrm{~cm}$ de comprimento da segunda válvula, na face anti-mesocólica, que foi fixada e estudada.

Nos grupos 1, 2 e 3 observou-se na serosa e nas camadas musculares, leve infiltrado inflamatório de células predominantemente mononucleares, principalmente próximo às suturas, onde foram também encontradas neoformação fibrovascular e reação granulomatosa do tipo corpo estranho ao fio de sutura, com presença de células gigantes.

Essa resposta inflamatória foi mais intensa no grupo 3, causando fibrose, presente em toda a área seccionada da serosa e das camadas musculares, separando-as. Evidenciou-se um anel fibroso, que determinou diminuição do lúmen intestinal, em resposta à cicatrização da serosa e das musculares invertidas pela sutura, propiciando a formação de uma válvula artificial. Não foi possível afirmar se houve hipertrofia muscular na extremidade proximal dos fragmentos analisados. A análise histopatológica também não revelou, qualitativamente, quaisquer alterações nos plexos mioentéricos dos três grupos de estudo (Figura 2, 3).

Observaram-se nas camadas mucosa e submucosa de todas as amostras, focos inflamatórios mono e polimorfonucleares, mais exuberantes no grupo 1, correspondendo a processo inflamatório de transição inicial entre uma fase aguda e crônica. Reação do tipo corpo estranho (com esboço de granulomas e raras células gigantes), associada ao fio de sutura, também foi identificada, juntamente com neoformação fibrovascular discreta, caracterizando fibrose leve, circunscrita, nos grupos 2 e 3 .

Em todos os casos avaliados pela histopatologia, observouse a presença de microabscessos nas camadas musculares e submucosa, que acompanharam as reações de células gigantes (Figura 4).

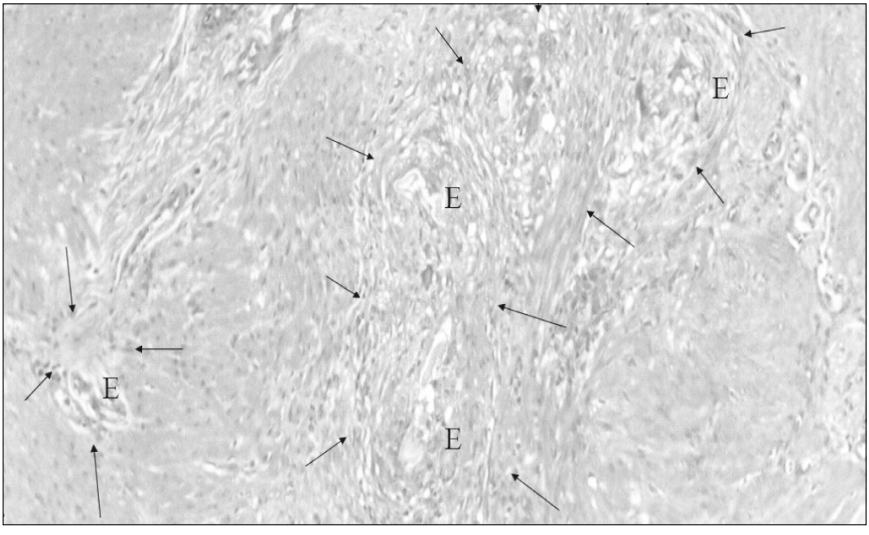

FIGURA 2 - Intestino - corte histológico de submucosa e muscular, apresentando: infiltrado inflamatório predominantemente mononuclear, esboço de formação de granulomas (em áreas confluentes) com células gigantes raras e corpo estranho associado (fio de sutura) (H-E x200) - Grupo 2 - Observação por 20 dias. (E: corpo estranho; setas: granulomas)

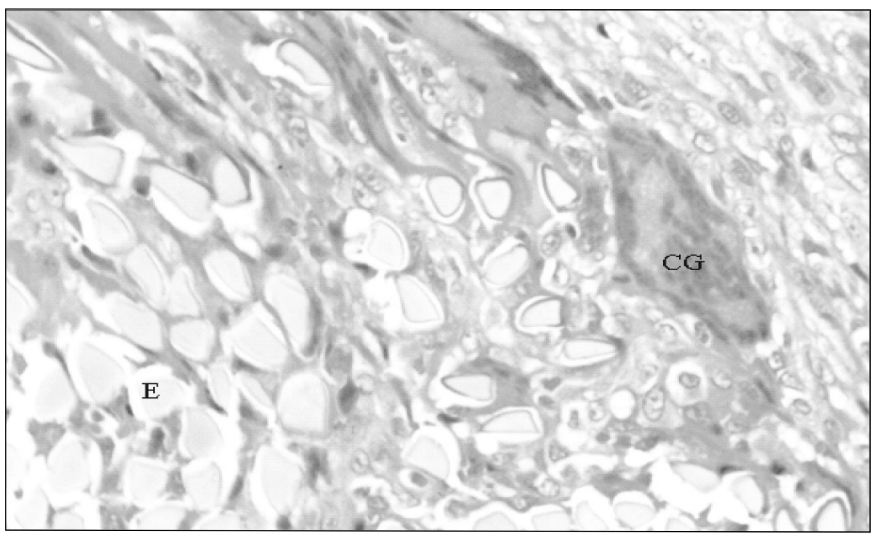

FIGURA 3 - Detalhe da reação giganto-celular a corpo estranho birrefringente (fio de sutura) - inflamação crônica granulomatosa (H-E x400) - Grupo 2 - Observação por 20 dias. (CG: célula gigante do tipo corpo estranho; E: corpo estranho)

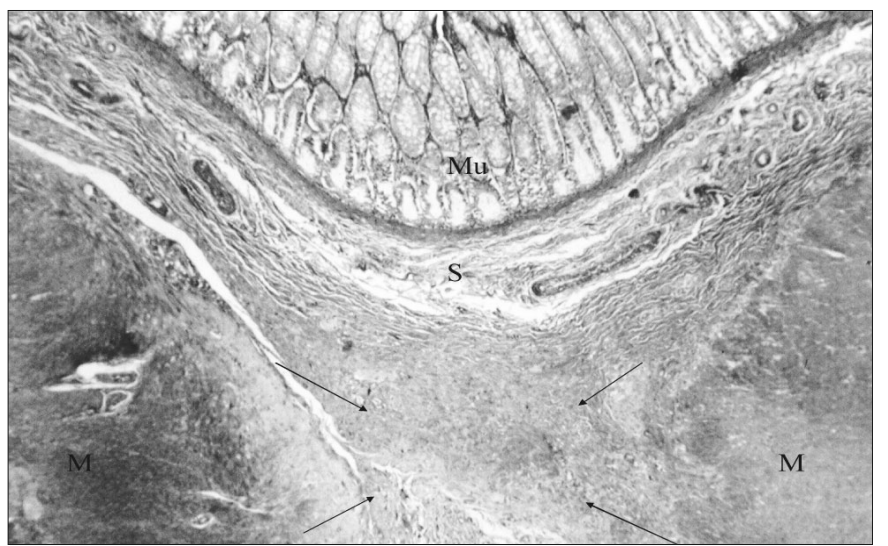

FIGURA 4 - Intestino - corte histológico, apresentando mucosa, submucosa e muscular. Área de neoformação fibrovascular entre as fibras musculares (setas), particularmente a camada interna (fibras colágenas coradas em verde) (tricrômio de Gomori x100) - grupo 2 - Observação por 20 dias. (Mu: mucosa; S: submucosa; M: muscular) 


\section{DISCUSSÃO}

Por muitos anos, a inquietude do cirurgião em obter um estado de continência para os doentes submetidos a ressecção do reto-ânus, com colostomia perineal, em casos de neoplasia maligna reto-anal, tem sido estímulo para constantes estudos em busca de melhores técnicas operatórias que proporcionem a substituição do esfíncter anal.

Alguns autores ${ }^{(4,5,24)}$ descreveram técnicas de transposição do músculo glúteo máximo para o tratamento da incontinência fecal e após amputação abdominoperineal, com bons resultados de continência.

Na segunda metade do século 20, idealizou-se a substituição do esfíncter anal por músculos longos da coxa ${ }^{(15,19,20,26)}$, sendo escolhido o músculo grácil, por sua facilidade de dissecação subcutânea com preservação de seu pedículo neurovascular.

A graciloplastia é operação que expõe o paciente a maior trauma e não resulta numa excelente taxa de continência, sendo necessário, por muitas vezes, o uso de estimuladores elétricos ${ }^{(30)}$ para manter a contração do músculo grácil, com função de esfíncter.

Nos anos 60, surgiram as operações de interposição de segmentos de intestino delgado, bem como a realização de miectomias com o objetivo de impedir a incontinência, retardando o trânsito intestinal ${ }^{(6)}$.

TEXTER $^{(27)}$ realizou miectomia circular segmentar no íleo terminal de cães, com exérese circunferencial da camada muscular longitudinal, produzindo diminuição no fluxo do conteúdo intestinal. Observou que as miectomias inibiram o trânsito intestinal. Esses resultados foram comprovados por outros autores ${ }^{(6)}$.

Propôs-se a criação de um esfíncter ileal, retirando-se as duas camadas musculares intestinais, circunferencialmente, com $1,5 \mathrm{~cm}$ de largura, ao invés de apenas a longitudinal ${ }^{(9)}$.

SCHMIDT, em 1982, apresentou estudo histológico, eletromicroscópico, manométrico e eletromiográfico da técnica de esfincteroplastia em colostomia. Usou músculo liso intestinal envolvendo o colo na região proximal à colostomia abdominal, tornando-a continente. Outros autores tiveram bons resultados com essa técnica ${ }^{(21,22,23,28)}$.

O índice percentual de continência pela técnica de Schmidt é muito bom porém, após sua realização, os enteroclismas são freqüentemente, necessários para a evacuação.

LÁZARO-da-SILVA ${ }^{(10,11,12,13)}$, unindo a radicalidade com o objetivo de evitar a colostomia abdominal, passou a fazer a colostomia no períneo. Aplicou seu método operatório de abaixamento abdominoperineal, com colostomia perineal valvulada. Construiu três válvulas acima do ponto de peritonização na parte alta da pelve, ainda com o colo sem fixação, pela da miotomia circular e longitudinal, invaginando a mucosa liberada, realizando-se sutura seromuscular. Entre as muitas vantagens relatadas pelo autor, algumas chamaram a atenção: evita-se a colostomia abdominal, permite exérese mais ampla dos tecido pélvicos, tornando a operação mais oncológica, a reparação se faz em torno de um quarto do tempo, a secreção da mucosa cólica é pequena na estomia, porque há oclusão parcial e pequena exteriorização do colo na pele; sob o ponto de vista social, oferece ao paciente melhores condições de convívio social do que com o uso da bolsa e há continência relativa. $O$ autor conclui, porém, que se tratando de proposição nova, seria necessário ainda estudar: se as válvulas teriam função retentiva, se a oclusão, por si, seria mecanismo parcial controlador da exoneração e que os resultados sob revisão não autorizavam conclusões definitivas e promissoras. Os pacientes, porém, preferem esse método à colostomia definitiva na parede abdominal.

A eficácia da construção de válvulas cólicas após a ressecção retoanal foi testada posteriormente em ratos. Evidenciou-se nas necropsias impacção fecal. Reforçaram a hipótese de que a seromiotomia age como mecanismo frenador, diminuindo o fluxo do trânsito intestinal ${ }^{(29)}$.

Seguindo o tratamento cirúrgico para a neoplasia retoanal proposto por MILES ${ }^{(16)} \mathrm{em} \mathrm{1908,} \mathrm{o} \mathrm{presente} \mathrm{trabalho} \mathrm{baseou-se}$ na investigação bibliográfica de suas modificações.

Os estudos pesquisados introduzem, após a amputação do reto-ânus, a colostomia perineal, com artifícios técnicos que provocam estase fecal. As evacuações são controladas já no início do pós-operatório tardio por esse procedimento, tentando-se uma continência. Além de ser tratamento para o câncer retoanal, causa trauma físico-psíquico e propicia desvantagens sociais, com a marginalização do indivíduo nos âmbitos familiar, conjugal, sexual, higiênico, além da falta de controle na eliminação de flatos e exoneração de fezes ${ }^{(13,14,25,29)}$.

A amputação abdominoperineal com estomia colocada no períneo e a associação dessa com a seromiotomia extramucosa tem mostrado excelentes resultados. Proporcionaram maior comodidade para as manipulações e melhor e adequada higiene, com ótima aceitação dos pacientes. As evacuações podem ser programadas pelas irrigações (enteroclismas). Muitas vezes, eles mesmos têm o controle do ato de evacuar, sabendo o dia provável da exoneração. A pelve fica preenchida pelo segmento do intestino grosso abaixado, o que permite o mecanismo de prensa abdominal que auxilia nas evacuações. A técnica é de fácil execução pelo cirurgião experiente, sendo de baixo custo, sem a necessidade de próteses ou de bolsas coletoras ${ }^{(13,14)}$.

\section{DISCUSSÃO DOS RESULTADOS}

Não houve diferenças significativas quanto ao controle morfológico das fezes entre os animais do grupo 1 e os do grupo-controle $1(P>0,05)$. Aconteceu porque, no período de 10 dias, o intestino grosso dos animais já estava se adaptando a sua nova fisiologia. A drenagem mucosa foi copiosa e constante, sendo sua produção aumentada pela perda vultosa e a falta de um esfíncter.

Nos animais acompanhados por 20 dias, os resultados mostraram haver diferenças significativas entre o grupo $2 \mathrm{e}$ grupo-controle $2(P<0,05)$, em relação ao último dia de controle morfológico das fezes líquidas. Para os animais do grupo 2, esse número foi significativamente inferior ao do grupo-controle 2 $(P=0,00387)$. Portanto, os mesmos apresentaram fezes líquidas por menos tempo que os cães do grupo-controle 2 , não se obtendo explicação para esse fato.

Nos animais acompanhados por 30 dias, existem diferenças significativas $(P<0,05)$, entre os grupo 3 e o grupo-controle 3 , no que se refere ao grau do controle morfológico das fezes $(P=0,0047)$. 
Na comparação entre os três grupos tratados (com válvulas) em relação à evolução do controle morfológico das fezes, houve diferença significativa $(P<0,05)$ entre os grupos, somente no que tange à variável grau do controle morfológico das fezes. $\mathrm{O}$ grupo 1 apresentou grau do controle morfológico inferior aos outros dois grupos $(P=0,011)$. Além disso, o grupo 2 e o grupo 3 não diferiram significativamente entre si. Observa-se que com o maior número de dias de observação entre os cães dos grupos 1,2 e 3, nota-se exonerações mais consistentes e até mesmo sólidas, refletindo o papel frenador das válvulas.

Observou-se neoproliferação conjuntiva com fibrose em toda a área seccionada da serosa e das camadas musculares, separando-as. Evidenciou-se um anel fibroso com diminuição do lúmen intestinal, em resposta à cicatrização da serosa e muscular invertidas pela sutura, levando à formação da já referida válvula artificial. As análises histopatológicas não revelaram qualitativamente quaisquer alterações nos plexos mioentéricos dos três grupos estudados.
Reação inflamatória de células mononucleares e granulares foi observada nas camadas mucosa e submucosa de todas as amostras, sendo ela mais intensa no grupo 1, correspondendo à resposta inflamatória inicial entre uma fase aguda e crônica Reações do tipo corpo estranho (reações de células gigantes) ao fio de sutura e neoproliferação conjuntiva, causando fibrose circunscrita, também foram encontradas. As suturas, portanto, produziram importantes processos inflamatórios nas camadas mucosa e submucosa.

O estudo da colostomia perineal valvulada experimental em cães, em resumo, propiciou o seguinte:

1. em todos os cães que apresentaram evacuações com fezes sólidas, constatou-se dilatação intestinal a montante de cada válvula, evidenciando estase fecal,

2. o processo inflamatório fibrosante reparativo ocorreu em todas as válvulas, associado a reações do tipo corpo estranho e à estenose parcial do lúmen intestinal.

Siqueira SL, Lázaro-da-Silva A, Reis OAF, Fantauzzi RS, Silva Jr OM, Sales PGO. Study of artificial valves on left colon after rectal partial abdominoperineal resection with perineal colostomy, in dogs. Arq Gastroenterol 2005;43(2):125-131.

ABSTRACT - Background - Throughout time, perineal colostomy in abdominoperineal resection, as a way to avoid abdominal colostomy, was studied. Perineal colostomy associated with a slowing down mechanism for stool transit has been studied for many years with satisfactory results. Aim - The investigation of a colic valvoplasty in dogs which have undergone an abdominoperineal resection plus perineal colostomy, and the discussion of the results achieved in clinic and histopathologic analysis are the objective of this study. Material and Methods - The experimental model of this research studied the colon of dogs. Sixty five animals were operated and divided in five groups: 10 animals in the pilot group, 15 animals in control group and 40 animals in 3 other groups of observation with 10,20 and 30 days of postoperative care. The dogs underwent a rectal partial abdominoperineal resection with perineal colostomy associated to a circumferential extramucosal seromiotomy of the left colon (except in the control group). Results - Through postoperative and post-mortem observation it was possible to verify solid stool before the valves showing the efficiency of the mechanism in slowing it down. The histological analysis of the valves showed connective growth with fibrosis in the whole incised serous and muscular layers, separating them. A fibrous ring diminished the colon lumen in response to the artificial valve created by the scar that appeared in the inverted suture of the serous and muscular layers. The inflammatory fibrous repair process occurred in all valves, associated to foreign body type reactions and to partial stricture of the colon lumen. Conclusion - The circumferential seromiotomy produces a fibrous ring that provides stool retention on the descending colon.

HEADINGS - Colostomy. Rectum, surgery. Dogs. 


\section{REFERÊNCIAS BIBLIOGRÁFICAS}

1. Cavina E, Seccia M, Chiarugi M. Continenza di colostomia perineale dopo operazione di miles: neosfintere elettrostimolato. Boll Soc It Chir. 1985;6:3.

2. Cavina E, Seccia, M, Evangelista G. Construction of a continent perineal colostomy by using electrostimulated gracilis muscles after abdominoperineal resection: personal technique and experience with 32 cases. Ital J Surg Sci. 1987;17:305-14.

3. Chiotasso P, Schmidt L, Juricik M, Lasorthes F. Acceptation des stomies périnéales. Gastroenterol Clin Biol. 1992;16:200.

4. Chittenden AS. Reconstruction of anal sphincter by muscle slips from the glutei. Ann Surg. 1930;92:152-4.

5. Chetewood $\mathrm{CH}$. Plastic operation for restoration of the sphincter ani, with report of a case. Med Rec. 1902;61:529.

6. Didio LJA, Anderson MC. The sphincters of the digestive system 1968; Baltimore: Williams Wilkins; 1968. p.184.

7. Gamagami RA, Chiotasso $P$, Lazorthes F. Continent perineal colostomy after abdominoperineal resection: outcome after 63 cases. Dis Colon Rectum. 1999;42:626-30.

8. Lasser P, Dubet P, Guillot JM, Elias D. Pseudocontinent perineal colostomy following abdominoperineal resection: technique and findings in 49 patients. Eur J Surg Oncol. 2001;27:49-53.

9. Lázaro-da-Silva A. Tentativa de tratamento do "dumping" através de um "esfíncter" ileal. Rev Assoc Med Minas Gerais. 1974;1:32-3.

10. Lázaro-da-Silva A. Amputação abdômino-perineal com colostomia perineal. Rev Bras Coloproctol. 1991;11:105-8.

11. Lázaro-da-Silva A. Amputação abdômino-perineal com colostomia perineal. Prensa Med Argent. 1995;82:800-4.

12. Lázaro-da-Silva A. Abdominoperineal excision of rectum and anal canal with perineal colostomy. Eur J Surg. 1995;161:761-4

13. Lázaro-da-Silva A, Capobiango A, Mattos MP. Abdominoperineal excision of the rectum and anal canal with perineal colostomy. ABCD Arq Bras Cir Dig. 1995;10:51-5.

14. Lázaro-da-Silva A. Tratamento do câncer reto-anal. In: colostomia perineal. São Paulo; Atheneu; 1998

15. Ma S, Leu SY, Fang RH. Reconstruction of anorectal angle after abdominoperineal resection of rectum and anus - an animal model. Ann Plast Surg. 1989;23:519-22.

16. Miles WE. A method of performing abdominoperineal excision for carcinoma of rectum and the terminal portion of the pelvic colon. Lancet. 1908;2:1812-3.
17. Petroianu A. Aspectos éticos na pesquisa em animais. Acta Cir Bras. 1996;11:157-64.

18. Petroianu A. Pesquisa experimental. In: Petroianu A. Ética, moral e deontologia médicas. Rio de Janeiro: Guanabara-Koogan; 2000, p.185-90.

19. Pickrell K, Masters F, Georgiade N, Hoton C. Rectal sphincter reconstruction using gracilis muscle transplant. Plast Reconstr Surg. 1954;13:46-55.

20. Pickrell K, Georgiade N, Maguire C. Transplantation of gracilis muscle to construct a rectal sphincter. Am J Surg. 1955;90:721-6.

21. Schmidt E, Bruch HP. Dickdarmmuskelauto-transplantation zur Behandlung der incontinentia alvi. Z Kinderchir. 1979;22:187.

22. Schmidt E, Bruch HP, Greulich M. Kontinent colostomie durch freie transplantation autologer dickdarmmuskulatur. Chirurg. 1979;50:96.

23. Schmidt E. Autologe darmmuskel-transplantation. Langenbecks Arch Chir. 1980;352:415-8.

24. Schoemarker J. Un nouveau procede operatoire pour la reconstituion du sphincter anal. Semaine Med. 1909;27:160.

25. Silva Jr AL. Histopatologia da seromiotomia dupla e sutura seromuscular no cólon descendente de ratos [dissertação]. Belo Horizonte: Faculdade de Medicina da Universidade Federal de Minas Gerais; 2000. p.65.

26. Simonsen SO, Stolf NA, Aun F, Raia A, Habr-Gama A. Rectal sphincter reconstrution in perineal colostomies after abdominoperineal resection for cancer. Br J Surg. 1976;63:389-91.

27. Texter EC Jr. Motility in the gastrointestinal tract. JAMA. 1963;25:640-7.

28. Torres RA, González MA. Perineal continent colostomy. Report of a case. Dis Colon Rectum. 1988;31:957-60.

29. Veloso SG, Biet R, Rios AM. Eficácia da confecção de válvulas colônicas após ressecção retoanal em ratos. Rev Col Bras Cir. 2001;28:356-63.

30. Williams NS, Hallan RI, Koeze TH, Watkins ES. Restoration of gastrointestinal continuity and continence after abdominoperineal excision of the rectum using an electrically stimulated neoanal sphincter. Dis Colon Rectum. 1990;33:561-5. 